\title{
Research on Reconstruction of Edge for Three-Dimensional Model Using Behavior Restriction
}

\author{
Liu Yan-zhong ${ }^{1}$, Liu Yan-ju ${ }^{2 *}$ and Zhang Hong-lie ${ }^{3}$ \\ ${ }^{1}$ College of Computer and Control Engineering, Qiqihar University, Qiqihar, \\ China \\ 2 Computer Center, Qiqihar University, Qiqihar, China \\ *15146692464@163.com
}

\begin{abstract}
Based on the analysis of the radial basis function (RBF) implicit surface reconstruction algorithm, an adaptive behavior control constrained reconstruction algorithm is proposed including point selection, the local two surface constraints, behavior selection of control points, and the radius of influence identified key links in detail. The validity of the algorithm is demonstrated on examples and shortcomings of exited algorithm by comparing. Finally the direction of improvement are analyzed and pointed out in the future.
\end{abstract}

Keywords: behavior control, constraint, point clouds, geometric complexity

\section{Introduction}

The point clouds are usually very large. A lot of problems for the subsequent processing are brought for hundreds of thousands, millions, or even hundreds of millions data in general. Therefore, how to accurate processing of raw data efficiently becomes a very important problem in surface reconstruction. The operation is very difficult in practice. Several methods are adopted in current popular local processing to reduce the error [1]. In all kinds of algorithm for interpolation constraint [2] to the point clouds, due to repair the generated by radial basis function (RBF) algorithm of graphics quality[3] excellent, the advantages of non-uniform data and noise data [4], such as the ability to adapt to the incomplete data [5], it is worth in-depth study of the algorithm [6]. Due to the adoption of the biggest bottleneck of RBF interpolation reconstruction algorithm is its high computational [7] in large-scale data processing point of complexity and storage requirements [8], so finding a solution algorithm of low complexity, adaptability is still need to continuously explore, referring to the advantages of the several algorithms to the basis, put forward a based on the "behavioral constraints" edge reconstruction [9] algorithm.

\section{The Principle of the Algorithm}

The algorithm is based on the fact that each part of the actual object surface shape features are not the same, some regional dramatic changes have steep edge reconstruction requires the accurate description and some regional variation in flat, can use the characteristics of relatively simple in its description, so in order to improve the adaptability of the algorithm, consideration should be given to the point of the surface of the original geometric characteristics, namely "behavior characteristics". The basic principle of this algorithm is selected according to the complexity and density of point cloud model adaptive behavior of surface characteristics of symmetric behavior control points and determine its feature types, using cell decomposition principle of control points 
using two surface function of local local constraints on the point clouds in each act, using compactly supported RBF to reduce the computational complexity. The following detailed description of the algorithm.

\section{1. Surface Modeling}

Point set $p=\left\{p_{1}, p_{2}, \ldots, p_{n}\right\}$ for the unknown surface $\mathrm{S}$ the sampling points, the direction of law vector $N=\left\{n_{1}, n_{2}, \ldots, n_{N}\right\}$ at each point as the indicator point at the surface to contain only the coordinates of the point of information data, through partial least-squares method to estimate the normal vector value. The algorithm the purpose is to find the implicit function $y=s(x)$, the $S(x)=0$ contour surface constraint point set $P$. Implicit surface space will be divided into two parts, usually set $s(x)>0$, when the points are located at the inner side surfaces; $s(x)<0$, when the point is located outside of the surface. In this paper, the following sections of each point of the method to a $s(x)<0$ point $s(x)>0$ side.

Point set $C=\left\{c_{1}, c_{2}, \ldots, c_{M}\right\}$, where $M<N$, as the center of symmetry $P$ selected from the set of points, point corresponds to a local reconstruction results for each behavior control, implicit surface model used in this thesis is:

$$
s(x)=\sum_{c_{i} \in C}\left[g_{i}(x)+\alpha_{i}\right] \psi_{r_{i}}\left(\left\|x-c_{i}\right\|\right)
$$

Among them, $\psi_{r}(x)=\psi(x / r), \psi(x)$ for radial basis function. Because the points set of $C$ than the point set $P$ contains less points, thus reduce the complexity of solving. Constraints in the symmetric behavior control point $C_{i}$ neighborhood within $\left\{|| x-c_{i}||<r_{i}\right\}, s(x)$ on the local two function binded, and then through the $M$ interpolation equations to solve the undetermined coefficient $a_{i}$, i.e.:

$$
S\left(c_{i}\right)=0, \quad i=1,2, \ldots, M
$$

Note that, for a linear type system, $p$ is $\psi_{r}$ to form a normalized radial basis function set. Usually choose psi $\psi(x)$ function has a local scope as similar to the Gauss function, this paper uses the compactly supported RBF :

Eq (1) can be transformed into:

$$
\psi(x)=(1-x)^{4}+(4 x+1)
$$

$$
s(x)=\sum_{c_{i} \in C} \alpha_{i} \psi_{r_{i}}\left(\left\|x-c_{i}\right\|\right)+\sum_{c_{i} \in C} g_{i}(x) \psi_{r_{i}}\left(\left\|x-c_{i}\right\|\right)
$$

On the right side of global constraints for the type of the point set, while the left part indicates the local details of point set. As can be seen Eq. (4) of global constraint part and the cell decomposition method have similar form, form of decomposition algorithm for unit

$$
\sum_{c_{i} \in C} g_{i}(x) \psi_{r_{i}}\left(\left\|x-c_{i}\right\|\right)
$$

Among them, $\Psi_{r_{i}}\left(\left\|x-c_{i}\right\|\right)=\frac{\psi_{r_{i}}\left(\left\|x-c_{i}\right\|\right)}{\sum_{j} \psi_{r_{j}}\left(\left\|x-c_{j}\right\|\right)}$ is the unit of decomposition which is used to solve the model, cell decomposition is widely used in computational mechanics, using it can get high quality constraints of point cloud surface model, so the improved combined unit decomposition method. $\{\Psi(\square)\}$ is called the normalized RBF. The constrained non uniform data can show better performance. This algorithm is also adopted this form to obtain the final model:

$$
\sum_{c_{i} \in C} \alpha_{i} \Psi_{r_{i}}\left(\left\|x-c_{i}\right\|\right)+\sum_{c_{i} \in C} g_{i}(x) \Psi_{r i}\left(\left\|x-c_{i}\right\|\right)=0
$$

The surface model makes full use of the advantages of local cell decomposition by the constraint method, modified by compactly supported RBF interpolation behavior control point complete local details. Because the behavior control points less than the point 
clouds so fast solution.

\subsection{The Local Constraint}

'louds acquisition is usually obtained from the different angle measurement data mosaic made, than other regions in the spliced position near the point density, in order to enhance the adapt ability of algorithm can deal with non-uniform density data, should specify a parameter to reflect the local point density, density features using a characterization parameter $\left\{p_{j}\right\}_{j=1}^{k} \lambda_{i}$ point $p_{i}$ point the $\lambda_{i}$ is defined as follows:

$$
\lambda_{i}=\sum_{j=1}^{k}\left\|p_{i}-p_{j}\right\|^{2}
$$

Among them, $\left\{p_{j}\right\}^{k}{ }_{j=1}$ is K the point closest to the $P_{i}$ point.

For each behavior control point $c_{i}$, in the R neighborhood of $\left\{x \| x-c_{i}<r_{i}\right\}$, the local constraint function structure of $g(x)$ constraint point set P. Figure. 1 shows the two -dimensional case description.

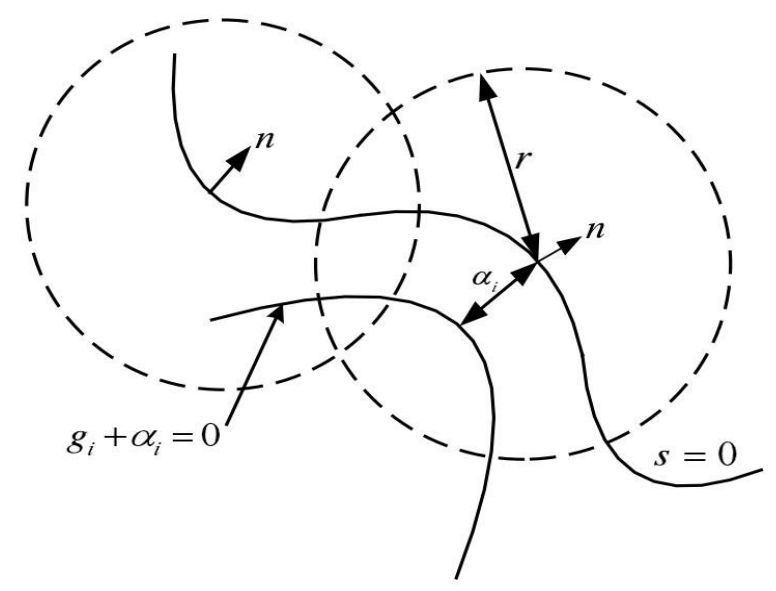

Figure 1. The Local Constraint Geometry Sketch

The behavior control points is defined local orthogonal coordinates $(m, K, l)$ for the first of all. Where $l$ is direction and $r$ is neighborhood average method to the same point, i.e.

$$
\sum_{j} \lambda_{j} \psi_{r}\left(\left\|p_{j}-c_{i}\right\|\right) n_{j}
$$

Among them, the unit normal vector $\mathrm{r}$ neighborhood $n_{j}$ for behavior control point $c_{i}$ point in $p_{i}$. Experimental results show that type of processing noise data is very effective. In the algorithm, the constrained function $g(x)$ using two surface:

$$
l=h(m, k) \equiv A m^{2}+2 B m k+C k^{2}+D m+E k+F
$$

Type through the following minimization process solution:

$$
\sum_{j} \lambda_{j} \psi_{r}\left(\left\|p_{j}-c_{i}\right\|\right) g_{i}\left(p_{j}\right)^{2} \rightarrow \min
$$

For a given $r$, the least squares problem type can be solved by orthogonal equation method.

Thus, $g_{i}(x)$ can be defined as:

$$
g_{i}(x)=l-h(m, k)
$$

Among them, $(m, K, l)$ for behavior control point $c_{i}$ local coordinate system, pay attention to the local constraint function $g(x)$ range depended on the local domain $r$. 


\subsection{Selection of Behavior Control}

As mentioned above, this algorithm was designed using a RBF constraint set, namely for the function $f(x)$ :

$$
f(x)=f(x)=\sum_{c_{i} \in C} \alpha_{i} \psi_{r_{i}}\left(\left\|x-c_{i}\right\|\right)+\sum_{c_{i} \in C} g_{i}(x) \psi_{r_{i}}\left(\left\|x-c_{i}\right\|\right)
$$

Among them, $c_{i} \subset P$ is the choice of radial basis function symmetric behavior control, embodies the absorption unit decomposed algorithm. Apparently reasonable selection of symmetric behavior control point, constructs the local constraint surface is the key unit, decomposition algorithm. Control point selection behavior principle, both to ensure support domain $\left\{\sup p \varphi\left(c_{i}\right)\right\}$ all behavior control point contains the entire set of points, also make symmetric behavior control points as little as possible. In addition, each region will have a certain overlap, and not less than a given threshold. Can be measured by the degree of overlap in parameter $o_{i} P_{i}$ :

$$
o_{j}=\sum_{j} \psi_{r_{i}}\left(\left\|p_{j}-c_{i}\right\|\right)
$$

The extent of overlap can be specified by the parameter $T_{p}$. In this paper, selection algorithm of behavior control point is described as follows:

Step a. Set up set overlap parameters of $O_{i}$ in each point of the $P_{i}$ in $P 0$

Step b. In point set randomly selected $K$, meet $O_{i}$ less than $T_{p}$

Step c. The selected $O_{i}$ minimum point in the Step b, this point as the RBF center $c_{m} \subset C$, and set the point $O_{m}=T_{p}$

Step d. Calculation of Step c as control point $C_{m}$ at the local two constraint function $g(x)$ and determine the support domain $r_{m}=r_{i}$

Step e. Through type (12) update the point set overlap parameter is located in the $C_{m}$ range of influence points in $P$

Step f. If the point of concentration is overlapped to a parameter $o_{i}<T_{p}$ point, return step b

Step g. End

\subsection{The Interpolation Coefficient}

In the local two surface constraint to complete each behavior control point, through the $M$ interpolation conditions (2)

Solving the coefficient $\alpha$, i.e.:

$$
s\left(c_{j}\right)=0=\sum_{c_{i} \in C}\left[g_{i}\left(c_{j}\right)+\alpha_{i}\right] \psi_{r}\left(\left\|c_{j}-c_{i}\right\|\right)
$$

Formula can be transformed into:

$$
\sum_{c_{i} \in C} \alpha_{i} \Psi_{i j}=-\sum_{c_{i} \in C} g_{i}\left(c_{j}\right) \Psi_{i j}, \Psi_{i j}=\psi_{r}\left(\left\|c_{j}-c_{i}\right\|\right)
$$

The compactly supported RBF using WENDALAND, so the Eq. (14) constitute a sparse linear system on $\alpha$. The compactly supported RBF is strictly positive definite, because the behavior of each point in the point set $C$ are different, so the components of the $M \times M$ interpolation matrix $\left\{\Psi_{i j}\right\}$ is positive definite. In this paper, using preconditioned conjugate gradient method for solving linear equations of Eq. (14) form, and set the initial value of $\alpha_{i}=0$.

\subsection{The Extraction of Implicit Surfaces}

The reconstruction of surface based on RBF is obtained for the implicit surface, its zero set is the desired. The implicit surface is obtained by ray tracing. However, in the practical application need to be implicit surface into the grid 
form, in order to facilitate the operation and storage. The extraction of implicit apply more type curve method for the marching cubes method or marching tetrahedra, which based on implicit surface $S$ is sampled, with a certain precision to obtain the surface of the polygon expression. To generate the vertices of the polygon are arranged according to a certain order, to ensure consistent method to each side direction and surface. Surface following algorithm adopted in this paper to extract the grid application more. The following is to introduce its principle.

Using the marching cubes extraction grid needs for space $N \times N \times N$ interval sampling, calculating function values at each grid point, its computational complexity is high up to $O(N)^{3}$, while the surface following algorithmonly needs to calculate the surface around the $\mathrm{S}$ value, the computational complexity is reduced to $O(N)^{2}$, when the function evaluation time consuming can be greatly to speed up the computation.

\section{Step of Algorithm and Program Design}

In this paper, the point cloud data processing algorithm for containing only $\mathrm{x}, \mathrm{y}$ and $\mathrm{z}$ coordinates of the data points, usually to save it to a certain format in the text file, the algorithm computes at each point normal vector and normalized, selection constraint control point and then adaptive and determine the radius of influence, and seek out part two surface constraint results, then solve the coefficient alpha, finally get the implicit surface reconstruction and mesh extraction result output. The algorithm for solving the structure is described as following.

Step 1. Input point cloud data

Step 2. The method of vector calculation

Step 3. Behavior center selection

Step 4. And to determine the local two times the radius of influence surface constraint

Step 5. If All points disposed

To solve the global interpolation coefficient

The hidden network type surface extraction output

Else

Return Step 3

Step 6. End

This paper realizes the algorithm using $\mathrm{C}++$ language, using object oriented programming method, the main steps of the algorithm in the abstract as the object, make clear thinking program design. Below is a brief description of the main program of:

Class Point Point is used to describe the point cloud elements and basic operations on it, the main data members and member function:

float (*point)[3]; // Storage point of X, y, Z coordinate array pointer

float (*normal)[3]; // The method of vector array pointer storage point

int point_num; // Point number

void SetPoint(int i, float $\mathrm{x}$, float $\mathrm{y}$, float $\mathrm{z}$ ) // Set the serial number for Ix, y, Z coordinates

void SetNormal(int i, float $\mathrm{x}$, float $\mathrm{y}$, float $\mathrm{z}$ ) //Set the serial number for $\mathrm{i}$

void AveragedNormal(float $n[3]$, int start, int end) //Average normal vector

void Centroid(float c[3], int start, int end) // Calculation of center point void Get Bound(float min[3], float $\max [3]$, int start, int end)

Class BasisFun is used to describe the radial basis function to solve, including RBF centers, support region, and local reconstruction two surface coefficient

float centerX, centerY, centerZ; // The radial basis function center coordinate

float $\mathrm{cXX}, \quad \mathrm{cYY}, \quad \mathrm{cZZ}, \quad \mathrm{cXY}, \quad \mathrm{cYZ}, \quad \mathrm{cZX}, \quad \mathrm{cX}, \quad \mathrm{cY}, \mathrm{cZ}, \mathrm{c0} ; / /$ two surface equation coefficient

float support; // Support domain radius

int level; // Hierarchy 
int index; // Radial basis function number

double value(float $\mathrm{x}$, float $\mathrm{y}$, float $\mathrm{z}$ ) //For function at a point value

void gradient(double $\mathrm{g}[3]$, float $\mathrm{x}$, float $\mathrm{y}$, float $\mathrm{z}$ ) // Compute the gradient at a point value

Class AdRbfGenerator is used to complete adaptive behavior control point selection, determination method of vector calculation of point cloud data, and calculate the radius of support key steps such as cutting plane, the main data members and member function:

PointSet* ps; // The pending point set

AxisK-dTree* tree; //K-d tree

int canN;// The specified parameter

float overlapT; // Specify the overlap parameter

float error; // Error

int bf_cnt; // Radial basis function count

BasisFunction** bf; //Radial basis function preservation solved

void generate() //The completion point clouds adaptive behavior control point selection and optimal selection of support domain

float localFit(float center[3], float scale, BasisFunction* bf) // Local fitting function

void global_solve() // Global fitting function, solving the coefficients of $\alpha$.

In addition, the program also defines some of the other classes, such as responsible for the output point cloud data reading and grid file to File Manager and is responsible for the matrix calculation mathematical model, because of the constraints.

\section{Reconstruction Examples Validate the Algorithm}

In this paper, the verification algorithm hardware platform for dual core $3.0 \mathrm{GHz}$, memory for $2 \mathrm{~GB}$. Figure 2 and 3 data taken from the HOPPE home page, in order to repair capacity verification algorithm for uneven density point cloud data and effect of reconstruction algorithm for incomplete data, point cloud data with the density change of the number and the structure light measurement method for data collection and reconstruction test. In Figure 2, the point clouds on the left side of the original data left to head is down sampled, the left point density is on the right side of the $1 / 6$, the scale of point cloud data for the $7.5 \mathrm{~K}$, set the error parameter is 0.001 , intermediate obtained for this algorithm to reconstruct behavior control, reduce $4.7 \mathrm{~K}$, implicit surfaces on the right rendering is obtained. You can see the algorithm according to the selection behavior of point cloud density change adaptive control point. Due to the left of point cloud data is sub sampled and the existence of missing details left reconstruction of surface.

In Figure 3, a) is a structure light measurement method of relief plates of point clouds, it can be seen, point distribution on a line, point density online, and have big distance between lines, $b$ ) for the reconstruction of the surface can be obtained using the presented algorithm, c) for surface single layer obtained by interpolation algorithm, although this algorithm can accurately reconstruct the object details, but the density variation in the relatively large holes appeared. It shows that the algorithm has a certain data repair capacity.
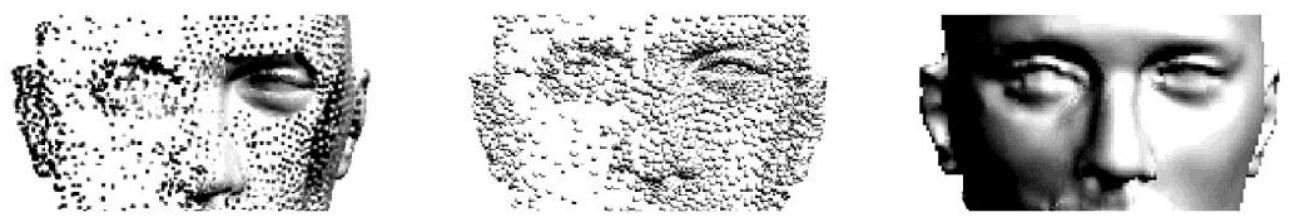

Figure 2. Density Changes of Data Reconstruction 


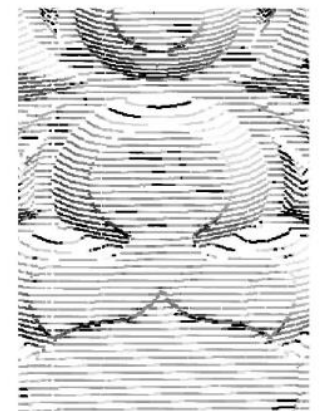

(a.)

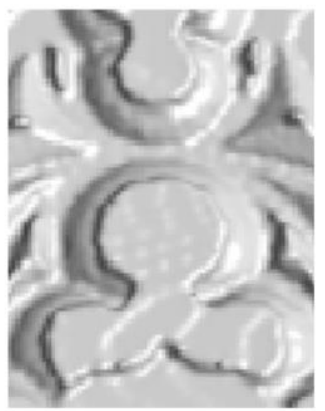

(b)

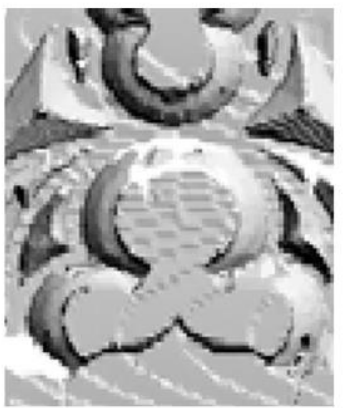

(c)

Figure 3. Structured Light Data Reconstruction

The key of this algorithm is to determine the behavior selection of control points and the corresponding radius of action, through the specified local error parameters for determining the radius of action, clear and easy to implement the algorithm idea is simple, good for uniform data and density change data reconstruction results, but by the experiment results also find it hard to part of the point clouds to find the error parameters the right to achieve the ideal effect of reconstruction algorithm, so the limitation of. The sparse constraint and MDL criterion is applied to the reconstruction algorithm, and achieved good results, in addition to the application of statistical technology related to point cloud surface reconstruction algorithm is also worth further research direction.

\section{Conclusions}

According to the direct use of RBF interpolation algorithm for solving the disadvantages of low efficiency, the difficulty, an adaptive behavior control point selection reconstruction algorithm is proposed that does not need the splicing operation segmentation and local solution of the point clouds after the given accuracy constraints. Local reconstruction is used to the non-interpolation and global one need not to add additional constraints. Therefore, the speed of reconstruction is increased. Experimental results show that the algorithm based on density of point clouds can determine the behavior control complexity adaptive. Even though the density changes, it also can get good results.

\section{Acknowledgments}

This work was jointly supported by the National Natural Science Foundation of China (Grant No. 61403222), Natural Science Foundation of Heilongjiang Province (Grant No. F201332, F201439, F201440).

\section{References}

[1] Y. Liu, N. Jing and L. Chen, “Algorithm for processing k-Nearest join based on R- Tree in map reduce,” Journal of Software, no. 8, (2013), pp. 1837- 1851.

[2] Y. J. Liu, Y. D. Zhang, B. R. Tao, H. L. Zhang and J. G. Jiang, "Progressive surface reconstruction algorithm for mass point clouds of denture," ICIC Express Letters, Part B: Applications, vol. 5 no. 5 , (2014), pp. 1273-1278.

[3] Y. J. Liu, Y. Z. Liu and B. R. Tao, "A novel self-organized fuzzy neural network surface reconstruction algorithm for point clouds without normal," International Journal of Database Theory and Application, vol. 7 no. 4, (2014), pp. 209-216.

[4] C. C. Kuo and H. T. Yau, "A Delaunay-based region-growing approach to surface reconstruction from unorganized points," Computer-aided Design, vol. 37 no. 8, (2005), pp. 825-835.

[5] W. H. Press, S. A. Teukolsky and W. T. Vetterling, "Numerical recipes: the art of scientific computing," Cambridge: Cambridge University Press, (2007), pp. 788-798. 
[6] H. M. Xia, "The radial basis function point cloud data surface reconstruction based on," Heilongjiang Science and Technology Information, no.2, (2010), pp. 42-44.

[7] J. Wang, Z. W. Yang and F. L. Chen, "A variational model for normal computation of point clouds," The Visual Computer, vol. 28 no. 2, (2012), pp. 163-174.

[8] K. Zhou, M. M. Gong and X. Huang, "Data parallel octree for surface reconstruction," IEEE Transactions on Visualization and Computer Graphics, vol. 17 no. 5, (2011), pp. 669-681.

[9] J. Rissanen, "Information and complexity in statistical modeling," New York: Springer, (2007), pp. 97-102. 